\title{
ON THE JOIN OF TWO COMPLEXES
}

\author{
C. E. CLARK
}

1. Introduction. In this note we point out an isomorphism between the $(r+1)$-dimensional Betti group of the join (defined below) of two complexes and a subgroup of the $r$-dimensional Betti group of the product of the two complexes. Using this isomorphism the Betti groups of the join are derived from those of the product in case the complexes are finite. ${ }^{1}$

2. Definition of the join $\left(K_{1}, K_{2}\right)$ of $K_{1}$ and $K_{2}$. To define the join of two complexes we first define the join $(\sigma, \tau)$ of a $p$-dimensional simplex $\sigma$ and a $q$-dimensional simplex $\tau, p, q=0,1, \cdots$. This join is a $(p+q+1)$-dimensional simplex with a $p$-dimensional side associated with $\sigma$ and the opposite side, which is $q$-dimensional, associated with $\tau$. These sides will not be distinguished from $\sigma$ and $\tau$, respectively. Now consider the complexes $K_{1}$ and $K_{2}$. Consider the set consisting of the simplexes $\sigma_{\alpha}$ of $K_{1}$, the simplexes $\tau_{\beta}$ of $K_{2}$, and the simplexes $\left(\sigma_{\alpha}, \tau_{\beta}\right)$. In a natural way this set forms a complex. We define the join $\left(K_{1}, K_{2}\right)$ of $K_{1}$ and $K_{2}$ to be the first barycentric subdivision of this complex.

3 . The rays. By the rays of $(\sigma, \tau)$ we mean the straight line segments each of which joins a point of $\sigma$ and a point of $\tau$. These rays cover $(\sigma, \tau)$. Also no two rays intersect except possibly at an end point. The rays of all $\left(\sigma_{\alpha}, \tau_{\beta}\right)$ of $\left(K_{1}, K_{2}\right)$ are called the rays.

Let $N_{i}, i=1,2$, be the subcomplex made up of the simplexes of $\left(K_{1}, K_{2}\right)$ that have at least one vertex in $K_{i}$ together with the faces of all such simplexes. It is known that each ray meets the intersection $N_{1} \cap N_{2}$ in exactly one point. ${ }^{2}$ Furthermore $N_{i}$ and $N_{1} \cap N_{2}$ can be homotopically deformed in $N_{i}$ along the rays into $K_{i}, i=1,2 .^{2}$ It follows that $N_{1} \cap N_{2}$ and the product $K_{1} \times K_{2}$ are homeomorphic (the complexes being considered as point sets).

4. The theorem. We prove this theorem.

THEOREM 1. There is an isomorphism between the $(r+1)$-dimensional

Received by the editors April 24, 1942.

1 The Betti groups of the join of two finite complexes are known. They were computed by H. Freudenthal in his paper Die Bettischen Gruppen der Verbindung Zweier Polytope, Fund. Math. vol. 29 (1937) pp. 145-150.

2 For a proof see our paper Simultaneous invariants of a complex and subcomplex, Duke Math. J. vol. 5 (1939) pp. 62-71. 
Betti group of $\left(K_{1}, K_{2}\right)$ and the subgroup of those homology classes of the $r$-dimensional Betti group of $N_{1} \cap N_{2}$ which contain cycles that bound both in $N_{1}$ and $N_{2}, r \geqq 0$; all chains considered in this paper are finite and have integral coefficients.

In this theorem $K_{1}$ and $K_{2}$ may be infinite.

Proof. We note that $\left(K_{1}, K_{2}\right)=N_{1}+N_{2}$. Hence we know that there is a homomorphism from the $(r+1)$-dimensional Betti group of $N_{1}+N_{2}$ on to those homology classes of the $r$-dimensional Betti group of $N_{1} \cap N_{2}$ which contain cycles that bound both in $N_{1}$ and $N_{2}, r \geqq 0$; furthermore, the kernel of this homomorphism consists of the homology classes that contain Summenzyklen, that is, cycles that are equal to the sum of two cycles, one in $N_{1}$ and the other ${ }^{3}$ in $N_{2}$. To prove Theorem 1 we shall show that a Summenzyklus bounds in $\left(K_{1}, K_{2}\right)$. To prove this, consider a cycle $Z$ of $N_{1}$. This cycle can be homotopically deformed in $N_{1}$ along the rays into a singular cycle of $K_{1}$. We know that $\left(K_{1}, K_{2}\right)$ contains the join of $K_{1}$ and an arbitrary point of $K_{2}$. Hence any cycle of $K_{1}$ can be homotopically deformed in $\left(K_{1}, K_{2}\right)$ into a cycle of a vertex of $K_{2}$. Since the dimension of $Z$ is greater than zero, it follows that $Z$ is homologous to zero in $\left(K_{1}, K_{2}\right)$. Similarly, a cycle of $N_{2}$ with dimension greater than zero bounds in $\left(K_{1}, K_{2}\right)$.

5. Some properties of $N_{1} \cap N_{2}$. From now on $K_{1}$ and $K_{2}$ are finite. For any chain $A$ of a complex let $|A|$ denote the closure of the set of those simplexes at which $A \neq 0$. We know that with each pair of chains $C_{1} \subset K_{1}$ and $C_{2} \subset K_{2}$ there is associated a chain $C_{1} \times C_{2} \subset N_{1} \cap N_{2}$; the dimension of $C_{1} \times C_{2}$ is the sum of the dimensions of $C_{1}$ and $C_{2}$; and $\left|C_{1} \times C_{2}\right|=\left|C_{1}\right| \times\left|C_{2}\right| .5$ It follows that the projection of $\left|C_{1} \times C_{2}\right|$ in to $K_{1}$ or $K_{2}$ along the rays is a subset of $\left|C_{1}\right|$ or $\left|C_{2}\right|$, respectively. Also if $C_{2}$ is a zero-dimensional cycle, and $C_{2}=0$ at all but one vertex of $K_{2}$ at which $C_{2}=1$, then $C_{1} \times C_{2}$ and $C_{1}$ are isomorphic, and homotopic deformation of $C_{1} \times C_{2}$ along the rays shows that $C_{1} \times C_{2} \sim C_{1}$ in $N_{1}$ (all the rays used in the deformation meet at a point of $K_{2}$ ).

Let

$$
Z_{i}^{p} \subset K_{1}
$$

${ }^{3}$ See Alexandroff-Hopf, Topologie. I, p. 293, Theorem V. This theorem and its applicability were pointed out by the referee.

4 An $r$-dimensional chain is a function defined over all $r$-dimensional simplexes of a complex.

${ }^{5}$ These and the following properties of $N_{1} \cap N_{2}=K_{1} \times K_{2}$ are proved in Alexandroff-Hopf, Topologie. I, pp. 299-310. 
and

$$
Z_{j}^{q} \subset K_{2}
$$

be homology bases ${ }^{6}$ for the $p$-dimensional cycles of $K_{1}$ and the $q$-dimensional cycles of $K_{2}$. We know that there is a set of $s$-dimensional chains $u_{k}^{s} \subset K_{1}, s=2,3, \cdots$, in $(1=1)$ correspondence with the $(s-1)$-dimensional torsion coefficients of $K_{1}$, and there is a set of $t$-dimensional chains $v_{l}^{t} \subset K_{2}, t=2,3, \cdots$, in $(1=1)$ correspondence with the $(t-1)$-dimensional torsion coefficients of $K_{2}$ such that a homology basis for the $r$-dimensional cycles of $N_{1} \cap N_{2}$ is given by

$$
Z_{i j}^{r}=Z_{i}^{p} \times Z_{j}^{q}, \quad p+q=r, \text { order of } Z_{i j}^{r} \neq 1,
$$

and

$$
C_{k l}^{r}=c_{k l}^{r}\left(u_{k}^{s} \times v_{l}^{t}\right)^{\cdot}, \quad s+t=r+1, c_{k l}^{r} \neq 1,
$$

where $c_{k l}^{r}$ is the reciprocal of the greatest common divisor of the torsion coefficients associated with $u_{k}^{s}$ and $v_{l}^{t}$. Furthermore the order of $Z_{i j}^{r}$ is the greatest common divisor of the orders ${ }^{7}$ of $Z_{i}^{p}$ and $Z_{j}^{q}$, and the order of $C_{k l}^{r}$ is the reciprocal of $c_{k l}^{r}$.

6. The cycles of $N_{1} \cap N_{2}$ that bound both in $N_{1}$ and $N_{2}$. We choose a cycle from (1) with $p=0$ and a cycle from (2) with $q=0$. Each of these cycles will be denoted by the same symbol $Z_{1}^{0}$.

Theorem 2. A homology basis for the subgroup mentioned in Theorem 1 consists of the following subset of (3) and (4): if $r>0$, the basis consists of $Z_{i j}^{r}$ with both $p>0$ and $q>0, Y_{i j}^{r}=Z_{i}^{r} \times\left(Z_{1}^{0}-Z_{j}^{0}\right), j \neq 1$, $X_{i j}^{r}=\left(Z_{1}^{0}-Z_{i}^{0}\right) \times Z_{j}^{r}$, $i \neq 1$, and all $C_{i j}^{r}$; if $r=0$, the basis consists of $W_{i j}^{0}=\left(Z_{1}^{0}-Z_{i}^{0}\right) \times\left(Z_{1}^{0}-Z_{j}^{0}\right), i \neq 1, j \neq 1$.

Proof. In the first place consider $Z_{i j}^{r}$ with both $p \neq 0$ and $q \neq 0$. We know that $Z_{i j}^{r}$ can be homotopically deformed in $N_{1}$ along the rays into $\left|Z_{i}^{p}\right|$, a $p$-dimensional subcomplex of $K_{1}$. Since $p<r$, this implies that $Z_{i j}^{r}$ bounds in $N_{1}$. From symmetry, if $p \neq 0$ and $q \neq 0$, the cycle $Z_{i j}^{r}$ bounds both in $N_{1}$ and $N_{2}$.

Consider next $C_{k l}^{r}$. Since $s+t=r+1, s>1$, and $t>1$, it follows that

${ }^{6} \mathrm{By}$ a homology basis for the $r$-dimensional cycles of a complex we mean a set of cycles obtained by expressing the $r$-dimensional Betti group as the usual direct sum of free cyclic groups and finite cyclic groups whose orders are the torsion coefficients and by choosing a cycle from a generator of each summand.

${ }^{7}$ By the order of a cycle we mean the order of its homology class. Also it is understood that a free group has order zero and that the greatest common divisor of zero and a positive integer is that integer. 
$s<r$ and $t<r$. Hence $C_{k l}^{r}$ bounds both in $N_{1}$ and $N_{2}$ because $C_{k l}^{r}$ is homotopic in $N_{1}$ to a cycle of $\left|u_{k}^{s}\right|$ and is homotopic in $N_{2}$ to a cycle of $\left|v_{l}^{t}\right|$.

Next consider $Z_{i j}^{r}$ with $p=r \neq 0$. We can assume that $Z_{j}^{0}=0$ at all but one vertex of $K_{2}$ and that $Z_{j}^{0}=1$ at the exceptional vertex. Then as shown above $Z_{i j}^{r} \sim Z_{i}^{r}$ in $N_{1}$. From this we shall deduce that $Z_{i j}^{r}$ does not bound in $N_{1}$. Suppose $Z_{i j}^{r}$ does bound in $N_{1}$. Then $Z_{i}^{r}=\dot{F}$, $F \subset N_{1}$. We deform $F$ in $N_{1}$ along the rays into the singular chain $F^{\prime} \subset K_{1}$. The singular cycle $Z_{i}^{r}=\dot{F}^{\prime}$ which contradicts the definition of $Z_{i}^{r}$.

From symmetry we see that $Z_{i j}^{r}$ with $q=r \neq 0$ does not bound in $\mathrm{N}_{2}$.

Now consider $Y_{i j}^{r}$. Since $Z_{i}^{r} \times Z_{1}^{0}$ and $Z_{i}^{r} \times Z_{j}^{0}$ are each homologous in $N_{1}$ to $Z_{i}^{r}$, it follows that $Y_{i j}^{r}$ bounds in $N_{1}$. That $Y_{i j}^{r}$ also bounds in $N_{2}$ follows from the fact that $Y_{i j}^{r}$ can be homotopically deformed into a complex consisting of two vertices. From symmetry we know that $X_{i j}^{r}$ bounds both in $N_{1}$ and $N_{2}$.

Finally if $r=0$, we see that $W_{i j}^{0}$ bounds both in $N_{1}$ and $N_{2}$.

The theorem follows easily from these facts.

7. The Betti groups of $\left(K_{1}, K_{2}\right)$. Theorems 1 and 2 imply this theorem.

FreUdenthaL's THEOREM. ${ }^{1}$ From (1) delete one cycle with $p=0$ and from (2) delete one cycle with $q=0$; each association of one of the remaining $Z_{i}^{p}$ with one of the remaining $Z_{j}^{q}, p+q=r$, represents a generator of the $(r+1)$-dimensional Betti group of $\left(K_{1}, K_{2}\right)$; the order of this generator is the greatest common divisor of the orders of $Z_{i}^{p}$ and $Z_{j}^{q}$; furthermore each $C_{k l}^{r}$ in (4) represents a generator of order $1 / c_{k l}^{r}$; all such generators with their orders define the $(r+1)$-dimensional Betti group of $\left(K_{1}, K_{2}\right)$.

8. The case $r=-1$. The join $\left(K_{1}, K_{2}\right)$ is connected because two points of $K_{i}$ can be joined to an arbitrary point of $K_{j}, j \neq i$.

Purdue University 